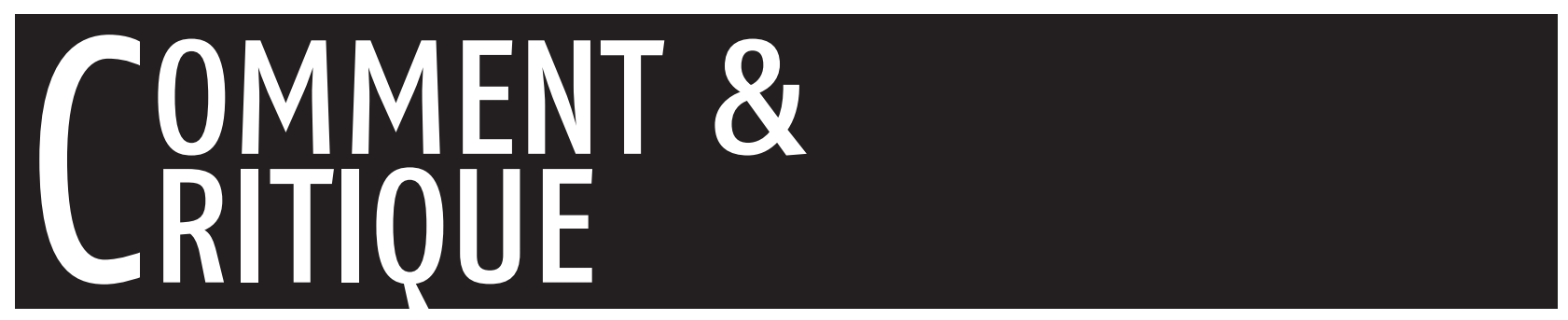

\section{Obsessive-compulsive disorder following left middle cerebral artery infarct}

Obsessive-compulsive disorder (OCD) is a syndrome characterized by recurrent, intrusive and inappropriate thoughts (obsessions) and repetitive and irrational behaviours (compulsions) (1). Although pathophysiologically heterogenous, anatomical sites such as orbital frontal lobe structures as well as the striatum and the basal ganglia region have been implicated for secondary OCD following head injury or temporal lobe epilepsy. We report a case of secondary OCD following a left middle cerebral artery infarct documented on magnetic resonance imaging (MRI) and single photon emission computed tomography (SPECT).

A 37-year-old man suffered a cerebral infarct in June 2002 involving the left middle cerebral artery territory. This episode was consecutive to acute sumatriptan abuse because of severe migraines. The patient was found to be hypertensive at that time. This infarct resulted in expressive aphasia and a right hemiplegia. The patient was treated in a rehabilitation centre for 5 months with satisfactory neurological improvement. Before discharge in October 2002, he developed OCD symptoms that included contamination obsessions, concerns with dirt and germs and compulsive washing. He washed his hands four to six times after each physical contact to such a degree that he had dermatological lesions. He tended to avoid personal contact. He brushed his teeth within $1 \mathrm{~h}$ after each meal. He acknowledged his own behaviours were excessive and his Yale-Brown Obsessive-Compulsive Scale score was 30 .

The patient had no history of any mental or physical illness except hypertension. Notably, he did not show any symptoms of depression, attention deficit or psychotic disorder. Physical and neurological examinations were normal with the exception of speech slowness, consecutive to the infarct, and this disappeared progressively.

Routine laboratory investigations, including thyroid function tests, serum folate and vitamin B12, were normal. On neuropsychological examination using the CERAD Neuropsychological battery and the Mattis Dementia Rating Scale, no impairment was found in memory or executive function or across other cognitive domains.

Brain MRI showed an infarct confined to the medial portion of the left temporal and parietal cortex. SPECT scan showed a global hypoperfusion of the left frontal, temporal and parietal regions, with the frontal region appearing to be less severely damaged.

He had no previous medication trial for OCD symptoms, and an ongoing trial of paroxetine $60 \mathrm{mg} /$ day and quetiapine 300 $\mathrm{mg}$ /day provided little relief. He was prescribed atenolol $50 \mathrm{mg} /$ day for hypertension.

The patient gave his consent for this comment to be published.

To our knowledge, this is the first case report of OCD following a left middle cerebral artery infarct. However, this kind of infarct is the most common of all cerebrovascular infarcts (2).

In this case, functional lesions in the frontal areas (SPECT) were more pronounced than in anatomical images (CT scan and MRI); this reminding us that this earlier brain imaging technique is better suited to detecting some brain lesions (3). Given the regions supplied by the middle cerebral artery, such as orbital surfaces and its anterolateral (thalamostriate) group of cerebral branches, which penetrate the caudate and lentiform nucleus, the areas damaged are those associated with obsessive-compulsive phenomena (4).

Moreover, frontal areas have an inhibiting function on human behaviours. Disinhibition caused by damage to the left orbito-frontal cortex (OFC) may have produced obsessive symptoms (5) in this patient.

Rational therapy for OCD must depend on a detailed understanding of the neuronal circuitry and physiological mechanisms underlying the patient's behaviours (3).

\section{Laurence Borras ${ }^{1}$ Benjamin Swine ${ }^{2}$ Eric-Louis Constant ${ }^{2}$ Philippe Huguelet ${ }^{1}$}

${ }^{1}$ Department of Psychiatry, Division of Adult Psychiatry, University Hospital of Geneva, 1207 Geneva, Switzerland; and

${ }^{2}$ Department of Psychiatry, Saint-Luc University Hospitals, Brussels, Belgium

Laurence Borras, MD Department of Psychiatry Division of Adult Psychiatry University Hospital of Geneva Consultation Secteur Eaux-Vives 36 Rue du XXXI Décembre, 1207 Geneva, Switzerland.

Tel: +4122 38231 03;

Fax: +4122 38231 05;

E-mail: laurence.borras@hcuge.ch

Acta Neuropsychiatrica 2007: 19:71-72

(C) 2007 The Authors

Journal compilation @ 2007 Blackwell

Munksgaard

DOI: $10.1111 / j .1601-5215.2007 .00178 . x$

\section{References}

1. American Psychiatric Association.

Diagnostic and statistical manual of mental disorders-DSM IV. Washington, DC:

American Psychiatric Association, 1994.

2. Hiano A. Examination of the brain. In: A guide to neuropathology, Tokyo: 


\section{COMMENT \& CRITIQUE}

Igaku-Shoin Ltd, chapter 2, 1993:

$10-92$.

3. Swoboda KJ, Jenike MA. Frontal abnormalities in a patient with obsessive-compulsive disorder: the role of structural lesions in obsessive-compulsive behavior. Neurology 1995;45:2130-2134.
4. Royall DR, Lauterbach EC, Cummings JL et al. Executive control function: a review of its promise and challenges for clinical research. A report from the Committee on Research of the American Neuropsychiatric Association. J Neuropsychiatry Clin Neurosci 2002;14:377-405.
5. Adler CM, McDonough-Ryan P, Sax KW, Holland SK, Arndt S, Strakowski SM. fMRI of neuronal activation with symptom provocation in unmedicated patients with obsessive compulsive disorder. J Psychiatr Res 2000;34: 317-324. 